\title{
Supporting information \\ Novel positive polaron stabilizing n-type host for high efficiency and long lifetime in blue phosphorescent organic light-emitting diodes
}

Do Sik Kim, Kyung Hyung Lee, Jun Yeob Lee*

School of Chemical Engineering, Sungkyunkwan University

2066, Seobu-ro, Jangan-gu, Suwon, Gyeonggi, 440-746, Korea

* Corresponding author

*E-mail:leej17@skku.edu,

\section{Experimental}

\section{General information}

All chemicals were purchased from chemical distributors or companies, with no further purification. 2,4-Dichlorobenzo[4,5]thieno[3,2,-d]pyrimidine was obtained from Samsung advanced institute of technology. 1,3-Dibromobenzene, 2-dicyclohexylphosphino-2',4',6'triisopropylbiphenyl (X-Phos), bis(pinacolato)diboron, palladium(II)acetate $\left(\mathrm{Pd}(\mathrm{OAc})_{2}\right)$, bis(pinacolato)diboron, $\quad$ [1,1'-bis(diphenylphosphino)ferrocene $]$ dichloropalladium(II) $\left(\mathrm{PdCl}_{2}(\mathrm{dppf})\right)$, and tetrakis(triphenylphosphine) palladium(0) $\left(\mathrm{Pd}\left(\mathrm{PPh}_{3}\right)_{4}\right)$ were purchased from $\mathrm{P} \& \mathrm{H}$ tech Co.. Triphenylsilyl chloride and n-butyllithium solution (n-BuLi) were supplied from Sigma Aldrich Co. Potassium carbonate and cesium carbonate used as a base were available from Samchun Chem. 1,2-Dichlorobenzene, 1,4-dioxane, tetrahydrofuran (THF), n-hexane (HEX), methylene chloride (MC), and methylbenzene (Tol) were purchased from Duksan Chemical Industry Co.. All reactions and manipulations were carried out under nitrogen condition. All liquid column chromatography (LC) was proceeded using silica gel as a stationary phase. Before device test, the final products secured purity of over $99.9 \%$ by sublimation purification after recrystallization.

The ${ }^{1} \mathrm{H}$ and ${ }^{13} \mathrm{C}$ nuclear magnetic resonance (NMR) spectra were obtained using a Unity Inova (Varian, 500, 300MHz) spectrometers. The HOMO and LUMO were calculated and estimated 
by a cyclic voltammetry (Ivium Tech., Iviumstat). The UV-vis spectra were obtained from UVvis spectrophotometer (JASCO, V-730) and the PL spectra were measured using a fluorescence spectrophotometer (PerkinElmer, LS-55). Both were performed using THF solution of the sample. The singlet and triplet energy values were measured at $77 \mathrm{k}$ using a frozen THF solution in liquid nitrogen. The instrument to measure mass values was Advion, Expresion ${ }^{\mathrm{L}}$ CMS spectrometer in APCI mode.

\section{Synthesis}

\section{(4-Bromophenyl)triphenylsilane}

(4-Bromophenyl)triphenylsilane was synthesized following the synthesis method from our previous paper. $^{27}$

\section{Triphenyl(4-(4,4,5,5-tetramethyl-1,3,2-dioxaborolan-2-yl)phenyl)silane}

Triphenyl(4-(4,4,5,5-tetramethyl-1,3,2-dioxaborolan-2-yl)phenyl)silane was also synthesized according to the synthesis method in our previous work. ${ }^{27}$

\section{4-(9H-carbazol-9-yl)-2-chlorobenzo[4,5]thieno[3,2-d]pyrimidine(1)}

2,4-Dichlorobenzo[4,5]thieno[3,2-d]pyrimidine (5.0 g, $11.9 \mathrm{mmol})$, 9H-carbazole (2.0 g, 11.9 mmol), 2-dicyclohexylphosphino-2',4',6'-triisopropylbiphenyl (1.15 g, $2.38 \mathrm{mmol})$, cesium carbonate $(7.75 \mathrm{~g}, 23.8 \mathrm{mmol})$ and bis(pinacolato)diboron, palladium(II)acetate $(0.79 \mathrm{~g}, 3.57$ mmol) were stirred in toluene $(50 \mathrm{~mL})$ at $110{ }^{\circ} \mathrm{C}$ for $6 \mathrm{~h}$ under a nitrogen condition. Subsequently, thin film chromatography was used to confirm the end of the reaction. The resulting mixture was extracted with distilled water and MC four times and then the organic solvent portion was separated. The crude product was purified by liquid column chromatography using an eluent of MC : $\operatorname{HEX}(1: 1)$ mixture. As a result, a light yellow product 
was obtained.

Yield 76.2\% (3.49 g), ${ }^{1} \mathrm{H}$ NMR (300 MHz, $\left.\mathrm{CDCl}_{3-} \mathrm{d}_{1}\right): \delta 8.64(\mathrm{~d}, 1 \mathrm{H}, \mathrm{J}=7.0 \mathrm{~Hz}), 8.15(\mathrm{~d}, 2 \mathrm{H}$, $\mathrm{J}=7.0 \mathrm{~Hz}), 7.85(\mathrm{~d}, 1 \mathrm{H}, \mathrm{J}=8.0 \mathrm{~Hz}), 7.74(\mathrm{t}, 3 \mathrm{H}, \mathrm{J}=7.0 \mathrm{~Hz}), 7.64(\mathrm{t}, 1 \mathrm{H}, \mathrm{J}=7.0 \mathrm{~Hz}), 7.51-7.40(\mathrm{~m}$, $4 \mathrm{H})$.

\section{4-(9H-carbazol-9-yl)-2-(3-(triphenylsilyl)phenyl)benzo[4,5]thieno[3,2-d]pyrimidine}

\section{(CzBTPmSi)}

4-(9H-carbazol-9-yl)-2-chlorobenzo[4,5]thieno[3,2-d]pyrimidine $\quad(3.0 \quad \mathrm{~g}, \quad 7.77 \mathrm{mmol})$, triphenyl(4-(4,4,5,5-tetramethyl-1,3,2-dioxaborolan-2-yl)phenyl)silane (3.96 g, $8.55 \mathrm{mmol})$, $\mathrm{Pd}\left(\mathrm{PPh}_{3}\right)_{4}(0.27 \mathrm{~g}, 0.23 \mathrm{mmol})$ and powdered potassium carbonate $(2.15 \mathrm{~g}, 15.55 \mathrm{mmol})$ were stirred in THF $(20 \mathrm{~mL})$ containing distilled water $(10 \mathrm{~mL})$ at $80{ }^{\circ} \mathrm{C}$ for $5 \mathrm{~h}$ under a nitrogen condition. The work-up and purification processes were the same as those of $4-(9 \mathrm{H}$-carbazol9-yl)-2-chlorobenzo[4,5]thieno[3,2-d]pyrimidine. As a result, a yellowish white product was obtained.

Yield 41\% (2.21 g), ${ }^{1} \mathrm{H}$ NMR (500 MHz, $\left.\mathrm{CDCl}_{3-} \mathrm{d}_{1}\right): \delta 9.08$ (s, 1H), 8.80 (d, 1H, J=8.0 Hz), $8.57(\mathrm{~d}, 1 \mathrm{H}, \mathrm{J}=7.5 \mathrm{~Hz}), 8.17-8.16(\mathrm{~m}, 2 \mathrm{H}), 7.83(\mathrm{~d}, 1 \mathrm{H}, \mathrm{J}=7.5 \mathrm{~Hz}), 7.75(\mathrm{~d}, 1 \mathrm{H}, \mathrm{J}=7.5 \mathrm{~Hz})$, 7.69-7.68 (m, 3H), 7.66-7.64 (m, 6H), 7.63-7.57 (m, 2H), 7.45-7.42 (m, 3H), 7.41-7.36 (m, 10H) MS (APCI) m/z $685.91\left[(\mathrm{M}+\mathrm{H})^{+}\right]$.

${ }^{13} \mathrm{C}$ NMR $\left(125 \mathrm{MHz}, \mathrm{CDCl}_{3}\right): \delta 162.37,161.56,153.11,141.80,139.02,138.87,136.96$, $136.69,135.06,134.33,133.83,131.30,129.93,129.87,128.54,128.15,126.33,125.66$, $125.22,124.67,123,20,122.71,121.97,120.54,112.81$.

\section{2-Chloro-4-(3-(triphenylsilyl)phenyl)benzo[4,5]thieno[3,2-d]pyrimidine(2)}

2,4-Dichlorobenzo[4,5]thieno[3,2-d]pyrimidine (5.0 g, $11.9 \mathrm{mmol})$, triphenyl(4-(4,4,5,5tetramethyl-1,3,2-dioxaborolan-2-yl)phenyl)silane (6.06 g, $13.1 \mathrm{mmol}), \mathrm{Pd}\left(\mathrm{PPh}_{3}\right)_{4}(0.41 \mathrm{~g}$, S-3 
$0.36 \mathrm{mmol})$ and powdered potassium carbonate $(3.29 \mathrm{~g}, 23.8 \mathrm{mmol})$ were stirred in THF (40 $\mathrm{mL})$ containing distilled water $(20 \mathrm{~mL})$ at $80{ }^{\circ} \mathrm{C}$ for $5 \mathrm{~h}$ under a nitrogen condition. The workup and purification processes were the same as those of 4-(9H-carbazol-9-yl)-2chlorobenzo[4,5]thieno[3,2-d]pyrimidine. As a result, a white product was obtained.

Yield 54.9\% (3.63 g), ${ }^{1} \mathrm{H}$ NMR (300 MHz, $\left.\mathrm{CDCl}_{3}-\mathrm{d}_{1}\right): \delta 8.56(\mathrm{~d}, 1 \mathrm{H}, \mathrm{J}=8.0 \mathrm{~Hz}), 8.39(\mathrm{~s}, 1 \mathrm{H})$, $8.32(\mathrm{~d}, 1 \mathrm{H}, 8.0 \mathrm{~Hz}), 7.83(\mathrm{~d}, 2 \mathrm{H}, \mathrm{J}=7.5 \mathrm{~Hz}), 7.69-7.58(\mathrm{~m}, 9 \mathrm{H}), 7.49-7.41$ (m, 9H).

\section{2-(9H-carbazol-9-yl)-4-(3-(triphenylsilyl)phenyl)benzo[4,5]thieno[3,2-d]pyrimidine $(\mathbf{m S i B T P C z})$}

2-Chloro-4-(3-(triphenylsilyl)phenyl)benzo[4,5]thieno[3,2-d]pyrimidine $\quad(3.0 \mathrm{~g}, 5.4 \mathrm{mmol})$, 9H-carbazole (0.9 g, 5.4 mmol), 2-dicyclohexylphosphino-2',4',6'-triisopropylbiphenyl (0.51 g, $1.08 \mathrm{mmol})$, cesium carbonate $(3.52 \mathrm{~g}, 10.8 \mathrm{mmol})$ and bis(pinacolato)diboron, palladium(II)acetate $(0.04 \mathrm{~g}, 0.16 \mathrm{mmol})$ were stirred in toluene $(40 \mathrm{~mL})$ at $110{ }^{\circ} \mathrm{C}$ for $6 \mathrm{~h}$ under a nitrogen condition. The work-up and purification processes were the same as those of 4-(9H-carbazol-9-yl)-2-chlorobenzo[4,5]thieno[3,2-d]pyrimidine. As a result, a yellowish white product was obtained.

Yield 33.2\% (1.23 g), ${ }^{1} \mathrm{H}$ NMR (500 MHz, $\left.\mathrm{CDCl}_{3-} \mathrm{d}_{1}\right)$ : 9.04 (d, 2H, J=8.5 Hz), 8.67 (d, 1H, $\mathrm{J}=7.5 \mathrm{~Hz}), 8.60(\mathrm{~s}, 1 \mathrm{H}), 8.48(\mathrm{~d}, 1 \mathrm{H}, \mathrm{J}=8.0 \mathrm{~Hz}), 8.11(\mathrm{~d}, 2 \mathrm{H}, \mathrm{J}=7.5 \mathrm{~Hz}), 7.88-7.84(\mathrm{~m}, 2 \mathrm{H})$, 7.71-7.68 (m, 8H), 7.64-7.61 (m, 1H), 7.47-7.45 (m, 5H), 7.43-7.36 (m, 8H) MS (APCI) m/z $685.91\left[(\mathrm{M}+\mathrm{H})^{+}\right]$.

${ }^{13} \mathrm{C}$ NMR $\left(125 \mathrm{MH}_{\mathrm{Z}}, \mathrm{CDCl}_{3}\right): \delta 160.64,160.57,157.00,142.76,139.67,139.20,137.20$, $136.71,136.14,133.88,133.63,131.06,130.24,130.06,129.01,128.32,126.78,125.89$, $125.58,124.80,123.27,123.24,122.17,119.74,116.32$.

\section{2,4-Di(9H-carbazol-9-yl)benzo[4,5] thieno[3,2-d]pyrimidine (2CzBTP)}


2,4-Dichlorobenzo[4,5]thieno[3,2-d]pyrimidine (5.0 g, $11.9 \mathrm{mmol})$, 9H-carbazole (4.38 g, 26.2 mmol), 2-dicyclohexylphosphino-2',4',6'-triisopropylbiphenyl (2.27 g, $4.76 \mathrm{mmol}$ ), cesium carbonate (11.63 g, $35.7 \mathrm{mmol})$ and bis(pinacolato)diboron, palladium(II)acetate $(0.79 \mathrm{~g}, 3.57$ mmol) were stirred in toluene $(60 \mathrm{~mL})$ at $110{ }^{\circ} \mathrm{C}$ for $8.5 \mathrm{~h}$ under a nitrogen condition. The work-up and purification processes were the same as those of 4-(9H-carbazol-9-yl)-2chlorobenzo[4,5]thieno[3,2-d]pyrimidine. As a result, a yellow product was obtained.

Yield 46.7\% (2.87 g), ${ }^{1} \mathrm{H}$ NMR (500 MHz, $\left.\mathrm{CDCl}_{3}-\mathrm{d}_{1}\right): 9.22(\mathrm{~d}, 2 \mathrm{H}, \mathrm{J}=8.0 \mathrm{~Hz}), 8.77(\mathrm{~d}, 1 \mathrm{H}$, $\mathrm{J}=7.5 \mathrm{~Hz}), 8.22(\mathrm{~d}, 2 \mathrm{H}, \mathrm{J}=7.0 \mathrm{~Hz}), 8.13(\mathrm{~d}, 2 \mathrm{H}, \mathrm{J}=7.5 \mathrm{~Hz}), 7.88(\mathrm{~d}, 1 \mathrm{H}, \mathrm{J}=8.0 \mathrm{~Hz}), 7.84(\mathrm{~d}, 2 \mathrm{H}$, $\mathrm{J}=7.5 \mathrm{~Hz}), 7.76-7.68(\mathrm{~m}, 2 \mathrm{H}), 7.53-7.40(\mathrm{~m}, 8 \mathrm{H}),\left(\mathrm{MS}(\mathrm{APCI}) \mathrm{m} / \mathrm{z} 516.61\left[(\mathrm{M}+\mathrm{H})^{+}\right]\right.$.

${ }^{13} \mathrm{C}$ NMR $\left(125 \mathrm{MH}_{z}, \mathrm{CDCl}_{3}\right): \delta 162.72,156.89,153.14,142.37,139.64,138.87,133.56$, $131.62,127.07,126.45,126.28,125.81,125.37,124.82,123.45,122.71,122.29,120.66$, $119.74,119.02,117.40,113.10$.

\section{2,4-Bis(3-(triphenylsilyl)phenyl)benzo[4,5]thieno[3,2-d]pyrimidine (2mSiBTP)}

2,4-Dichlorobenzo[4,5]thieno[3,2-d]pyrimidine $(3.0 \mathrm{~g}, 7.14 \mathrm{mmol})$, triphenyl(4-(4,4,5,5tetramethyl-1,3,2-dioxaborolan-2-yl)phenyl)silane (7.60 g, $16.4 \mathrm{mmol}), \mathrm{Pd}\left(\mathrm{PPh}_{3}\right)_{4}(0.25 \mathrm{~g}$, $0.21 \mathrm{mmol})$ and powdered potassium carbonate $(2.96 \mathrm{~g}, 21.4 \mathrm{mmol})$ were stirred in THF (30 $\mathrm{mL})$ containing distilled water $(15 \mathrm{~mL})$ at $80{ }^{\circ} \mathrm{C}$ for $5.5 \mathrm{~h}$ under a nitrogen condition. The workup and purification processes were the same as those of 4-(9H-carbazol-9-yl)-2chlorobenzo[4,5]thieno[3,2-d]pyrimidine. As a result, a white product was obtained.

Yield 30.9\% (1.89 g), ${ }^{1} \mathrm{H}$ NMR (500 MHz, $\left.\mathrm{CDCl}_{3-} \mathrm{d}_{1}\right): 9.01(\mathrm{~s}, 1 \mathrm{H}), 8.70(\mathrm{~d}, 1 \mathrm{H}, \mathrm{J}=8.0 \mathrm{~Hz})$, $8.52(\mathrm{t}, 2 \mathrm{H}, \mathrm{J}=8.5 \mathrm{~Hz}), 8.34(\mathrm{~d}, 1 \mathrm{H}, \mathrm{J}=8.0 \mathrm{~Hz}), 7.80(\mathrm{t}, 2 \mathrm{H}, \mathrm{J}=7.5 \mathrm{~Hz}), 7.74$ (d, 1H, J=7.0 Hz), 7.68-7.61 (m, 14H), 7.58-7.54 (m, 2H), 7.48-7.40 (m, 18H), (MS (APCI) m/z 855.20 [(M + $\left.\mathrm{H})^{+}\right]$.

${ }^{13} \mathrm{C}$ NMR $\left(125 \mathrm{MHz}_{2} \mathrm{CDCl}_{3}\right): \delta 161.18,160.15,158.97,142.11,138.95,138.45,137.56$, S-5 
$137.27,136.81,136.75,136.72,136.65,135.52,134.58,134.50,133.99,133.92,130.73$, $130.42,129.99,129.82,129.73,128.84,128.34,128.27,128.13,126.32,125.40,124.65$, 122.97.

\section{Device fabrication}

The device fabrication was proceeded using a device stack of indium tin oxide (ITO, 50 nm)/BPBPA:HATCN (40 nm:30 wt\%)/BPBPA (10 nm)/mCBP (10 nm)/mCBP:n-type host:CNImIr (30 nm, $50 \mathrm{wt} \%, 15 \mathrm{wt} \%) / \mathrm{DBFTRz}(5 \mathrm{~nm}) / \mathrm{ZADN}(20 \mathrm{~nm}) / \mathrm{LiF}(1.5 \mathrm{~nm}) / \mathrm{Al}(200$ nm). The N-type hosts were CzBTPmSi, mSiBTPCz, 2CzBTP and 2mSiBTP. The CNImIr doping concentration used in each emitting layer was $15 \mathrm{wt} \%$. The full names for the materials used for the layers other than the EML are as follows. The BPBPA is $N, N, N^{\prime} N^{\prime}$-tetra[(1, $1^{\prime}-$ biphenyl)-4-yl]-(1,1'-biphenyl)-4,4'-diamine, $\quad$ HATCN $\quad$ is $\quad 1,4,5,8,9,11$ hexaazatriphenylenehexacarbonitrile, $\mathrm{mCBP}$ is 3,3'-di(9H-carbazol-9-yl)-1,1'-biphenyl, DBFTRZ is 2,8-bis(4,6-diphenyl-1,3,5-triazin-2-yl)dibenzo[b,d]furan and ZADN is 2-[4-(9,10di-naphthalen-2-yl-anthracene-2-yl)-phenyl]-1-phenyl-1H-benzimidazole. All layers of the device structure were deposited by vacuum thermal evaporation under a high pressure of $4.0 \times$ $10^{-7}$ Torr. The device was encapsulated with a glass lid in a nitrogen-filled glove box to protect from moisture and oxygen. Electrical characterization of the devices was performed using a Keithley 2400 source meter and optical characterization was carried out using a CS 2000 spectroradiometer. The lifetime of the device was evaluated at an initial luminance of $100 \mathrm{~cd}$ $\mathrm{m}^{-2}$ under a constant current driving condition.

Hole and electron only devices were fabricated using a device stack of ITO (50 nm)/BPBPA:HATCN (40 nm:30 wt\%)/BPBPA (10 nm)/mCBP (10 nm)/n-type host(30 $\mathrm{nm}) / \mathrm{DNTPD}(10 \mathrm{~nm}) / \operatorname{Al}(200)$ and ITO $(50 \mathrm{~nm}) / \mathrm{n}$-type host(30 nm)/DBFTRz $(5 \mathrm{~nm}) / \mathrm{ZADN}$ $(20 \mathrm{~nm}) / \mathrm{LiF}(1.5 \mathrm{~nm}) / \mathrm{Al}(200 \mathrm{~nm})$, respectively. 
(a)

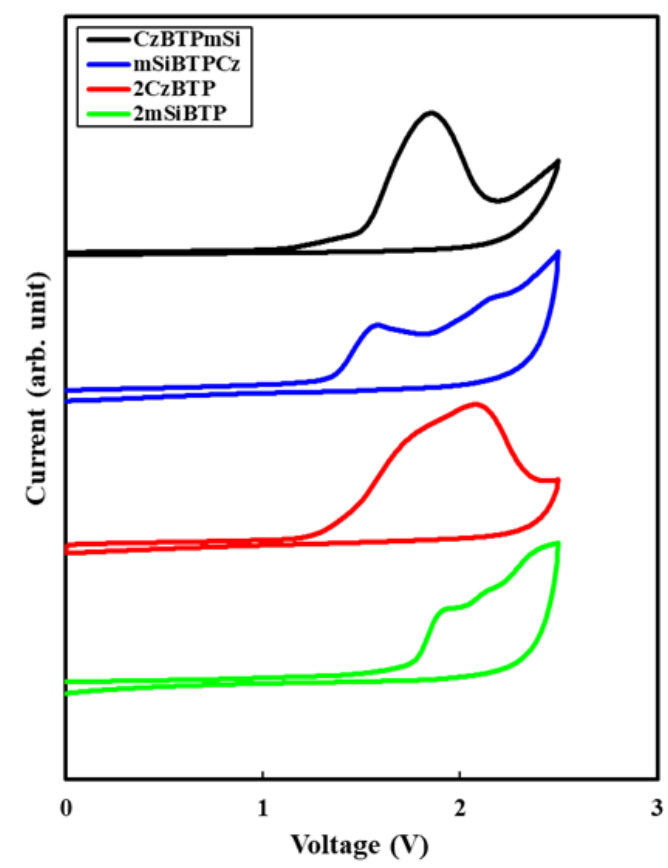

(b)

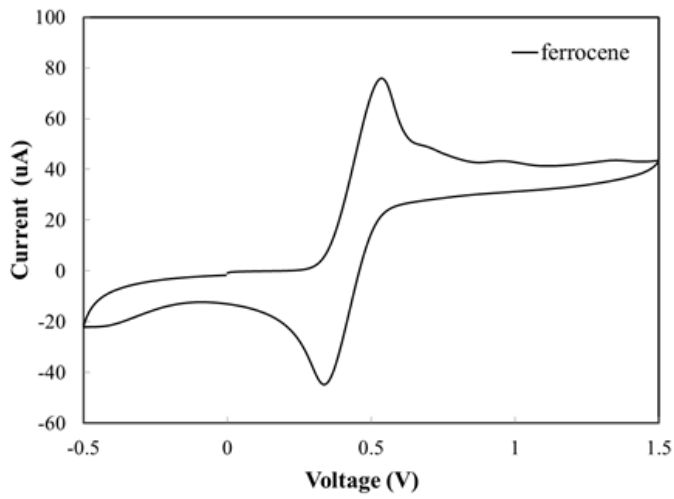

Figure S1. (a) The cyclic voltammetry scan of the CzBTPmSi, mSiBTPCz, 2CzBTP and 2mSiBTP n-type hosts and (b) the ferrocene standard. 
(a)

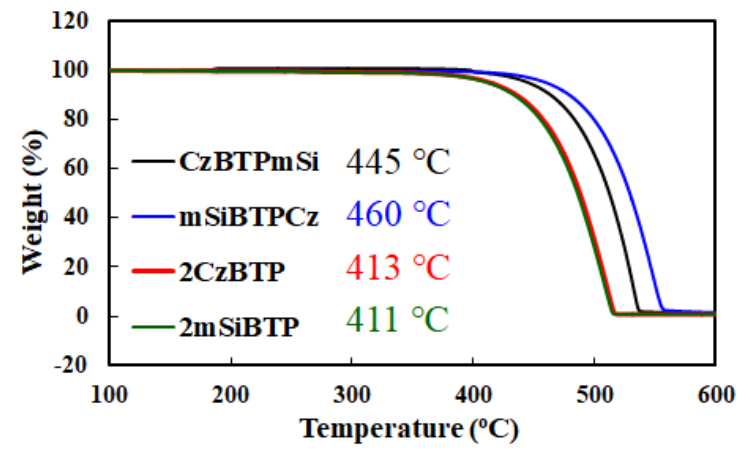

(b)

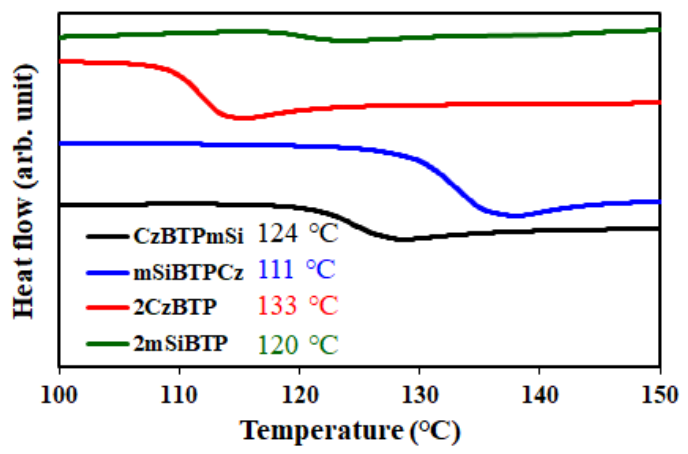

Figure S2. (a) TGA and (b) DSC of CzBTPmSi, mSiBTPCz, 2CzBTP and $2 \mathrm{mSiBTP}$ hosts at a heating rate of $10^{\circ} \mathrm{C} / \mathrm{min}$. 
(a)

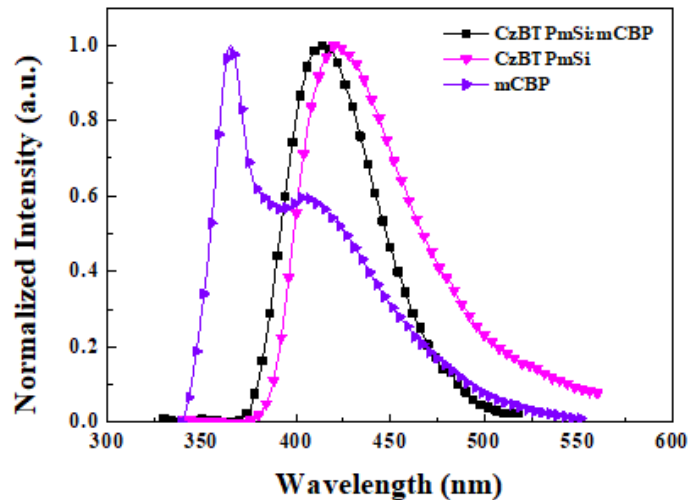

(c)

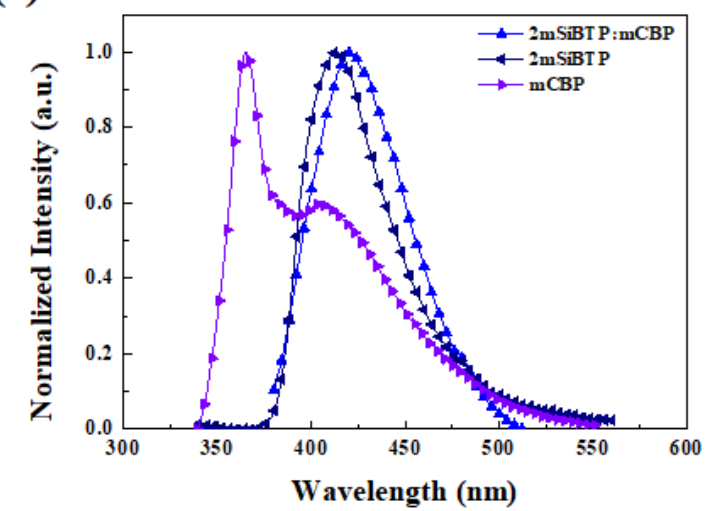

(b)

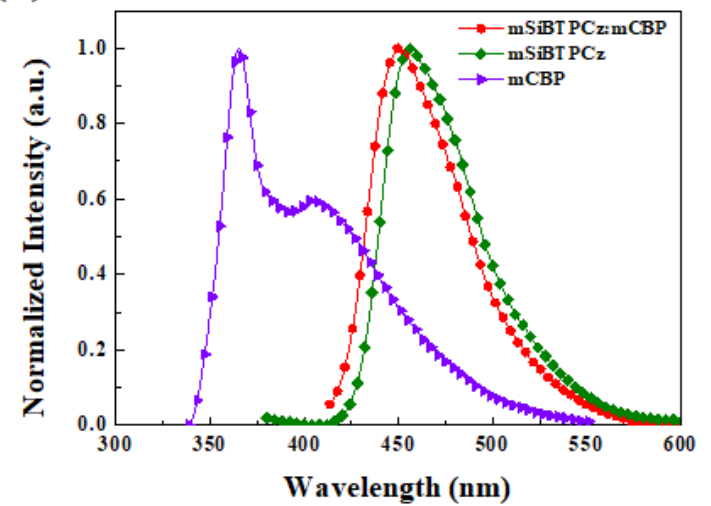

Figure S3. PL spectra of the host and mixed host films based on (a) CzBTP, (b) mSiBTPCz and (c) $2 \mathrm{mSiBTP}$. 
(a)

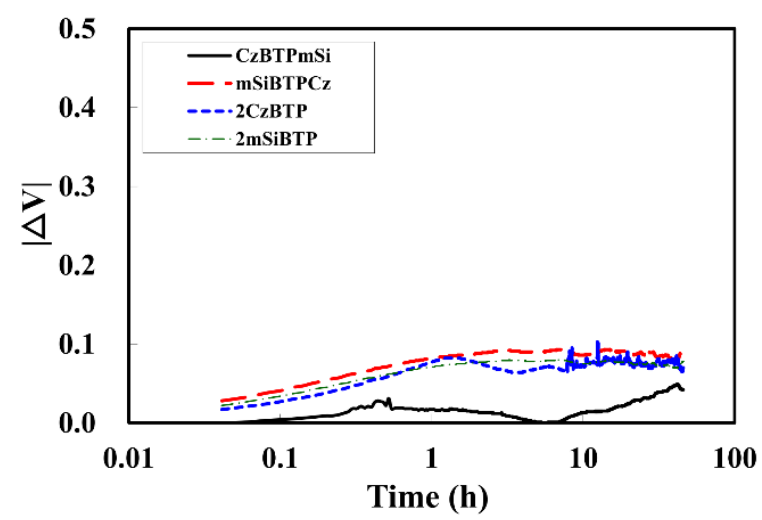

(b)

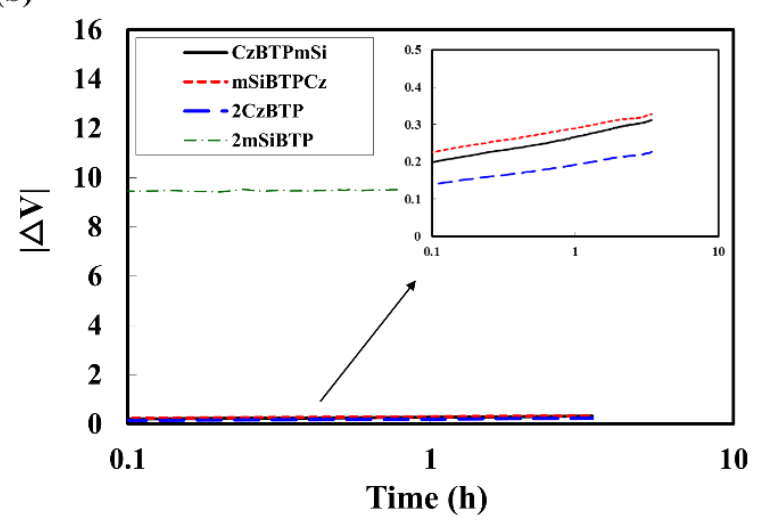

Figure S4. The voltage rise of the n-type hosts during electrical aging test of the electron only

(a) and hole only (b) devices. Current densities of the electron only and hole only device aging test were 75 and $5 \mathrm{~mA} \mathrm{~cm}^{-2}$. 
Table S1. Summarized the device performances data of the CNImIr and the $\operatorname{Ir}(\mathrm{cb}) 3$ doped CzBTPmSi:mCBP device at $1,000 \mathrm{~cd} \mathrm{~m}^{-2}$.

\begin{tabular}{cccccc}
\hline CNImIr (15 \%) & $\mathrm{CIE}^{\mathrm{a}}(\mathrm{x}, \mathrm{y})$ & $\begin{array}{c}\mathrm{EQE}^{\mathrm{b}} / \mathrm{EQE}_{\max } \\
(\%)\end{array}$ & $\begin{array}{c}\text { Lifetime }^{\mathrm{c}} \\
(\mathrm{h})\end{array}$ & $\begin{array}{c}\mathrm{PE}^{\mathrm{d}} / \mathrm{PE}_{\max } \\
(\mathrm{lm} / \mathrm{W})\end{array}$ & $\begin{array}{c}\mathrm{CE}^{\mathrm{e}} / \mathrm{CE}_{\max } \\
(\mathrm{cd} / \mathrm{A})\end{array}$ \\
\hline CzBTPmSi:mCBP & $(0.16,0.27)$ & $18.1 / 23.9$ & 70 & $16.7 / 33.9$ & $33.3 / 43.1$ \\
mSiBTPCz:mCBP & $(0.16,0.27)$ & $1.8 / 16.1$ & 41 & $1.3 / 30.1$ & $3.4 / 30.4$ \\
2CzBTP:mCBP & $(0.16,0.27)$ & $17.7 / 22.1$ & 21 & $17.1 / 31.8$ & $33.7 / 42.2$ \\
2mSiBTP:mCBP & $(0.16,0.28)$ & $16.2 / 21.6$ & 25 & $14.6 / 27.9$ & $30.5 / 40.9$ \\
mCBP & $(0.15,0.25)$ & $3.8 / 15.3$ & 6 & $2.3 / 23.8$ & $6.6 / 26.6$ \\
\hline Ir(cb)3 (20\%) & $\mathrm{CIE}^{\mathrm{a}}(\mathrm{x}, \mathrm{y})$ & $\mathrm{EQE}^{\mathrm{b}} / \mathrm{EQE}_{\max }$ & $\mathrm{Lifetime}^{\mathrm{c}}$ & $\begin{array}{c}\mathrm{PE}^{\mathrm{d}} / \mathrm{PE}_{\max } \\
(\mathrm{lm} / \mathrm{W})\end{array}$ & $\begin{array}{c}\mathrm{CE} / \mathrm{CE}_{\max } \\
(\mathrm{cd} / \mathrm{A})\end{array}$ \\
\hline CzBTPmSi:mCBP & $(0.13,0.17)$ & $15.3 / 19.9$ & 19 & $11.2 / 22.3$ & $20.3 / 26.6$ \\
\hline
\end{tabular}

a: color coordinate at luminance of $1,000 \mathrm{~cd} \mathrm{~m}^{-2}$, b: external quantum efficiency at $1,000 \mathrm{~cd} \mathrm{~m}^{-2}$, c: LT50, device lifetime at $1,000 \mathrm{~cd} \mathrm{~m}^{-2} \mathrm{~d}$ : power efficiency at $1,000 \mathrm{~cd} \mathrm{~m}^{-2}$, e: current efficiency at $1,000 \mathrm{~cd} \mathrm{~m}^{-2}$. 